\title{
Educación ciudadana en Colombia: dos coordenadas para un posible análisis
}

Citizen education in Colombia: two coordinates for a possible analysis

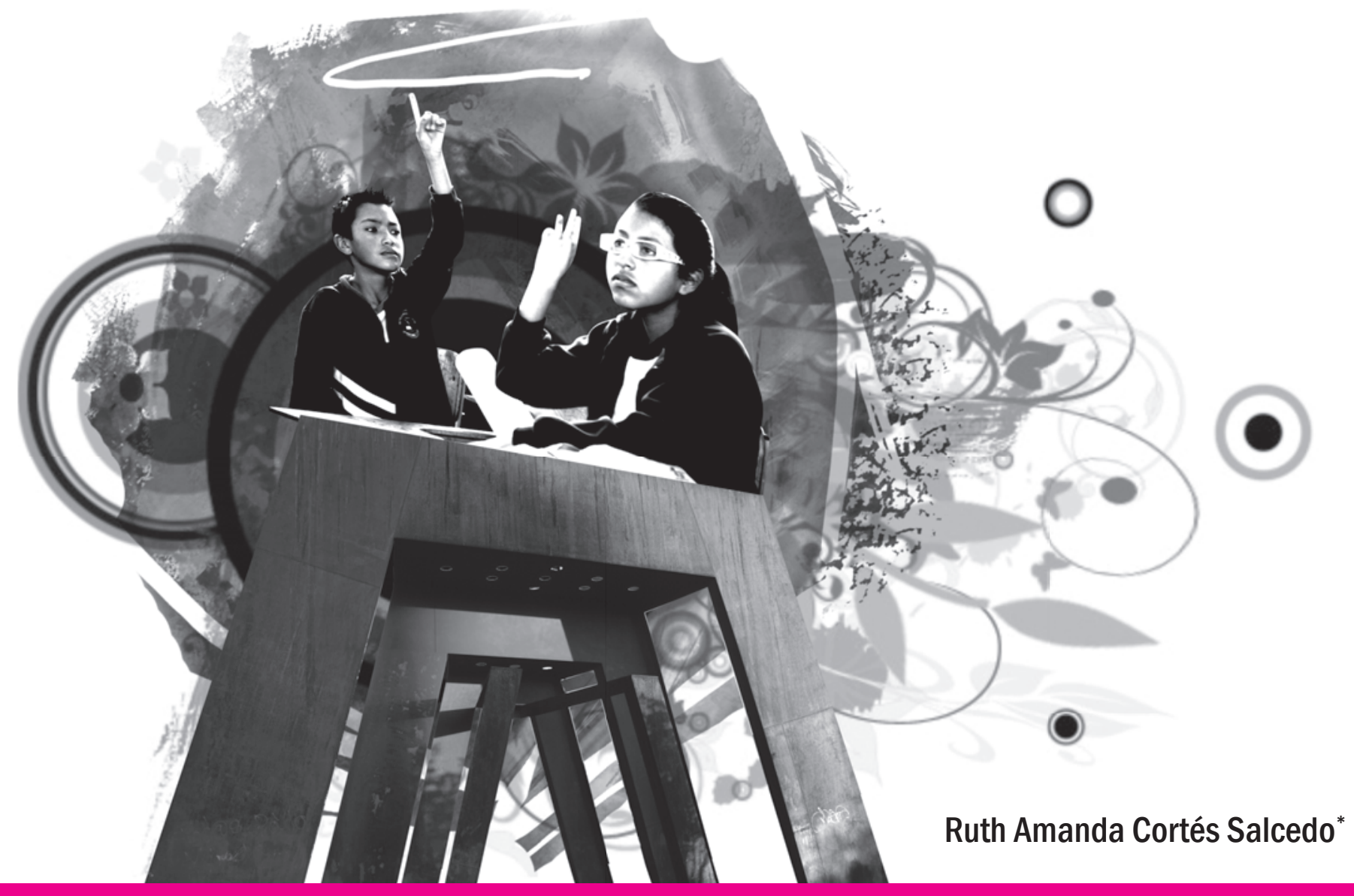

* Profesional del área académica del Instituto para la Investigación Educativa y el Desarrollo Pedagógico, IDEP. Especialista en Enseñanza de la Historia, Pontificia Universidad Javeriana; Magíster en Desarrollo Educativo y Social, Universidad Pedagógica Nacional, CINDE; Doctoranda en Educación, Universidad Pedagógica Nacional. 
Resumen El presente artículo pretende hacer una reseña bibliográfica crítica de dos estados del arte sobre la educación democrática y ciudadana en Colombia, con el objeto de mostrar cómo este tema se ha instalado en los últimos años en el campo de la investigación socioeducativa. Los dos estudios analizados amplían de manera complementaria una significativa masa documental que abarca diversas instituciones y organizaciones que producen discursos sobre educación ciudadana y muestran la pluralidad de los temas abordados y los énfasis según los intereses de quien los produce, constituyéndose en un importante aporte para quienes indagan sobre el tema.

Palabras claves: educación ciudadana, investigación educativa.

Abstract The present article tries to do a bibliographical critical review of two states of the art on the democratic and citizen education in Colombia to show as this theme has been established in the last years in the field of the socio-educative research. Both analyzed studies, extend in a complementary way a significant documentary mass that it includes diverse institutions and organizations that produce discourses on citizen education, show plurality of this, and the emphases according to the interests of that one who produces them, constituting itself in an important contribution for those who research on the subject.

Keywords: citizen education, educative research. 


\section{Presentación}

Abordar la cuestión de la educación ciudadana escolar en Colombia exige revisar la producción intelectual sobre el tema y realizar un balance crítico del mismo que permita identificar nuevas líneas de investigación, nuevas preguntas, o sencillamente, nuevas formas de mirarla.

En este artículo haremos referencia a dos estados del arte que por lo significativo del corpus documental trabajado, representan una síntesis de la producción intelectual sobre el trabajo empírico de la educación ciudadana en Colombia entre 1980 y 2006.

Como lo identificara en el año 2002 el Grupo de Investigación Representación, Discurso y Poder, de la Universidad Distrital, si bien algunos estudios desde la historia, la política, el derecho, la economía, la sociología y la antropología han trabajado las implicaciones de fenómenos políticos, económicos y educativos en la constitución de la ciudadanía y en su construcción en el universo de lo público, el tema de la ciudadanía sigue siendo marginal. De hecho un inventario general sobre estudios e investigaciones cuyo eje central lo constituya la ciudadanía deja percibir el poco desarrollo del tema en nuestro país, con excepción de los últimos diez años, en los cuales se evidencia una progresiva colonización de este tema por varias disciplinas (García Duarte et al., 2002, 15).

Derivado de la anterior afirmación, los estudios que tienen como tema central "la educación ciudadana" escolar son también escasos, con la misma excepción señalada por el Grupo. Sobre esta producción hay ya varios estados del arte y dos de ellos son los analizados a continuación. 


\section{De la educación democrática a la educación ciudadana}

El estado del arte realizado para Bogotá por Alexis Pinilla y Juan Carlos Torres (2006) titulado "De la educación democrática a la educación ciudadana: una década de incertidumbres", incluye otros siete estados sobre el tema, lo que muestra un alto grado de sofisticación analítica del asunto, en tanto que muestra una lectura de segundo nivel (un metaestado del arte) respecto de la producción intelectual sobre el trabajo empírico hecho especialmente en Bogotá.

El periodo delimitado por el estudio fue el de 1994 a 2004, y organizó la información y el análisis en dos ámbitos, uno: estados del arte sobre el tema, y dos: proyectos de innovación adelantados por las mismas instituciones escolares y proyectos de investigación sobre la ciudadanía y la formación ciudadana desarrollados

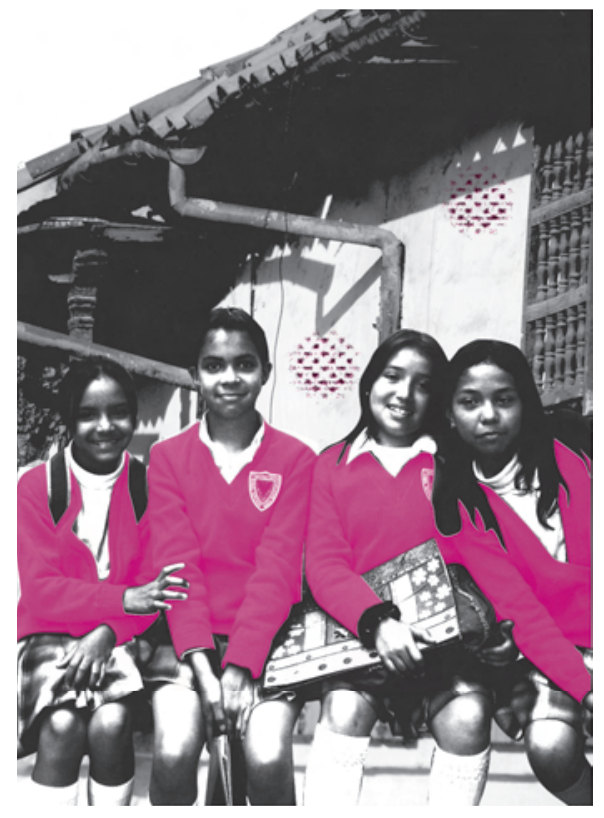
por universidades, centros de investigación, ONG y organismos estatales.

Los autores justifican el período por ser para ellos una característica fundamental de los años noventa, la centralidad que adquiere el tema de la ciudadanía en la agenda política y social de los Gobiernos en la mayoría de los países del mundo (Ibíd., p. 11), que se constata en los discursos internacionales, las agendas educativas, los análisis sobre el estado de la educación sobre todo en América Latina, y en investigaciones académicas. En Colombia esta característica es situada por los autores en los fundamentos de la Constitución Política de1991 y en la Ley General de Educación, donde el tema de la educación cívica y ciudadana se convirtió en uno de los ejes centrales de las políticas y los procesos educativos y, por extensión, de la sociedad en general. 
La metodología tuvo un carácter cualitativo, con un enfoque hermenéutico-interpretativo que presenta inicialmente un análisis del contexto sociopolítico y educativo de Colombia en la década de los noventa a partir de la siguiente tesis: el país se movió en una gran paradoja: la adopción simultánea de la Constitución Política de 1991 y del modelo económico neoliberal. Esa contradicción entre una apertura política y una apertura económica, con intereses y orientaciones divergentes, marcó todos los desarrollos legislativos posteriores, incluida la reforma educativa y las apuestas por la construcción de ciudadanía, tanto en las dirigidas a la ciudad como en las dirigidas a la educación.

El estudio muestra las políticas educativas de la década centradas en la evaluación y producción de estándares de educación ciudadana y las políticas urbanas, haciendo énfasis en los gobiernos de Bogotá, especialmente los de Antanas Mockus, quien explícitamente propuso estrategias para la formación ciudadana. Los estados del arte $^{1}$ analizados por los autores han hecho visible varios aspectos. En primer lugar, revelan cómo se ha ido configurando la educación ciudadana como un campo de discusión amplio y diverso. En segundo lugar, hacen visible la recurrencia de temas como los derechos humanos, la resolución de conflictos, la promoción de la paz, el poder, la convivencia, la cultura democrática escolar, la relación ciudad-educación, la educación moral, y los valores en contextos de violencia.
Finalmente, señalan una tendencia metodológica de corte cualitativo-etnográfico con énfasis en la descripción y la interpretación de las investigaciones propiamente dichas.

Cabe decir que quienes han construido estos estados del arte también exponen sus lugares teóricos desde donde leen la documentación. Se destacan perspectivas de la filosofía moral, la ética y la sociología desde donde trabajan categorías como racionalidad comunicativa y argumentativa, socialización política y cultura política.

Pinilla y Torres afirman que estos estados del arte constatan que en las investigaciones sobre educación ciudadana prima un carácter empírico-descriptivo que no logra abordar la complejidad de la realidad y del contexto de la escuela. Esta tendencia podría explicarse porque la mayor parte de los estados del arte trabajaron sobre programas de intervención y no sobre investigaciones, y por supuesto cabe la posibilidad de que el tema de la educación ciudadana sea una prioridad más de acciones políticas que de construcción de conocimiento académico. Así lo afirman Pinilla y Torres:
Hemos evidenciado que en esta materia hay un déficit de reflexión teórica y un exceso de realidad empírica, es decir, la necesidad de formar ciudadanos desde la escuela, motivada, en buena parte, por la crisis estructural de

1 Los siete estados de arte referenciados son: Rodríguez Álvaro (1995). Proyecto Educación Cívica. Revisión bibliográfica para el estudio nacional de caso en Colombia, Santa Fe de Bogotá, Ministerio de Educación Nacional. Mejía Marco Raúl y Restrepo Gabriel. (1997). Formación y educación para la Democracia en Colombia, Bogotá - Unesco, Instituto para el Desarrollo de la Democracia Luís Carlos Galán. Riaño Clara et al. (1999). Producción de conocimiento y prácticas pedagógicas en educación para la convivencia democrática desde la escuela, Bogotá, Colegio Santo Ángel - IDEP. Hoyos Guillermo. (2001). Formación ética, valores y democracia, Bogotá, Colciencias - Socolpe. Restrepo Gabriel. (2001). Estado del arte en las innovaciones e investigaciones en formación en valores en el Distrito de Bogotá, IDEP. Rodríguez Gregorio y Miñana Carlos. (2002). Formación ciudadana y urbana, Bogotá, Universidad Nacional y Herrera Martha et al. (2005). La construcción de cultura política en Colombia, Bogotá, Universidad Pedagógica Nacional. 
nuestro sistema socio-político, ha motivado [sic] que docentes, investigadores, administradores de la educación, directivos, etc., propongan diversas - y a veces divergentesrespuestas sobre el tema, las cuales parecen desbordar la capacidad analítica para teorizar la ciudadanía. En este sentido, a pesar de haber recorrido un camino bastante significativo en esta materia aún no tenemos certezas del significado de la ciudadanía escolar.

Los autores consultaron, además de los estados del arte, 36 proyectos de investigación e innovación, de los cuales 21 fueron apoyados por el IDEP y los 15 restantes financiados por Colciencias. ${ }^{2}$ Las investigaciones seleccionadas están circunscritas en el campo de la investigación educativa y fueron clasificadas en 3 ámbitos, así: a) Investigación en derechos humanos y para los derechos humanos, b) Resolución de conflictos y convivencia escolar, y c) Ética, educación moral y formación en valores. Estos últimos representaron la mayor tendencia de la masa documental trabajada por los autores.

El análisis de estas investigaciones muestra el recorrido en el campo de la educación ciudadana escolar. Por ejemplo, en el ámbito de los derechos humanos emergen categorías como género y desplazamiento; en el segundo, las categorías de conflicto, cultura democrática, paz, participación, multiculturalismo, y sus relaciones con el currículo y los espacios formales de participación escolar; el manual de convivencia aparece como objeto de estudio, así como el gobierno escolar y la figura del personero estudiantil desde la categoría de sujeto político o subjetividad política. En el tercer ámbito, las perspectivas de la filosofía y la psicología, principalmente, hacen visibles categorías como desarrollo moral, personalidad moral y comunidad justa. Los enfoques metodológicos en su mayoría son de corte cualitativo, etnográfico y hermenéutico.

Las principales conclusiones de Pinilla y Torres apuntan a situar el giro de la educación ciudadana en nuestro país a partir de la promulgación de la Constitución Política de 1991 y consideran que la proliferación de programas, investigaciones e innovaciones generaron ambigüedad en el uso del término "ciudadanía” y una yuxtaposición de concepciones sobre la misma que ellos evidencian en la inclusión de diversas estrategias reconocidas en los ámbitos descritos. Finalmente hacen una crítica a la realización de evaluaciones masivas y el establecimiento de estándares de competencias ciudadanas, las cuales consideran que, además de estandarizar el problema de la ciudadanía, reducen lo político básicamente a tres dimensiones: conocimientos sobre el Estado (ciudadano informado), actitudes positivas frente a la democracia (ciudadano virtuoso), y etapa del desarrollo moral en la cual se encuentran los niños (ciudadano racional)

Sobre las conclusiones de los autores habría que señalar varias cosas: la primera es en relación con la paradoja que enuncian en la tesis, lo que conduce a preguntarse si la economía y la política hoy día se oponen o más bien se han conjugado para organizar la sociedad de una manera particular. 
Sobre la proliferación de programas, investigaciones e innovaciones, podría interrogarse justamente las condiciones en las que se da esa explosión discursiva, las intenciones que llevan a hablar cada vez más de la ciudadanía y qué efectos se espera de ello.

Por otra parte, consideraríamos, contrario a los autores, que la ley no necesariamente funda una práctica sino que es parte de ella, por lo que habría que mirar la política de los estándares no en la forma unidireccional que configura acciones en la escuela por parte del maestro y de los estudiantes, sino que hay formas de apropiación que mostrarían matices en los modos como opera la educación ciudadana en la escuela, e incluso resistencias a ella.

Llama la atención que los autores afirmen que en relación con la formación ciudadana, incluso desde la década de los ochenta, se empezara a delegar en la escuela y en los docentes el discurso de cultura de paz, de los derechos, con lo que se intentaba democratizar la vida escolar y preparar a los ciudadanos para intervenir en el sistema democrático. Si bien el periodo estudiado es reciente, no se podría eludir la pregunta por la historicidad de la educación ciudadana escolar en Colombia, la cual podría mostrar que esa delegación tiene antecedentes que se remontan inclusive hasta el siglo XIX. Lo que habría que mirar es si ello es parte de un nuevo discurso o, por el contrario, es una continuidad que se desenvuelve en la escuela con sus propios ritmos culturales.

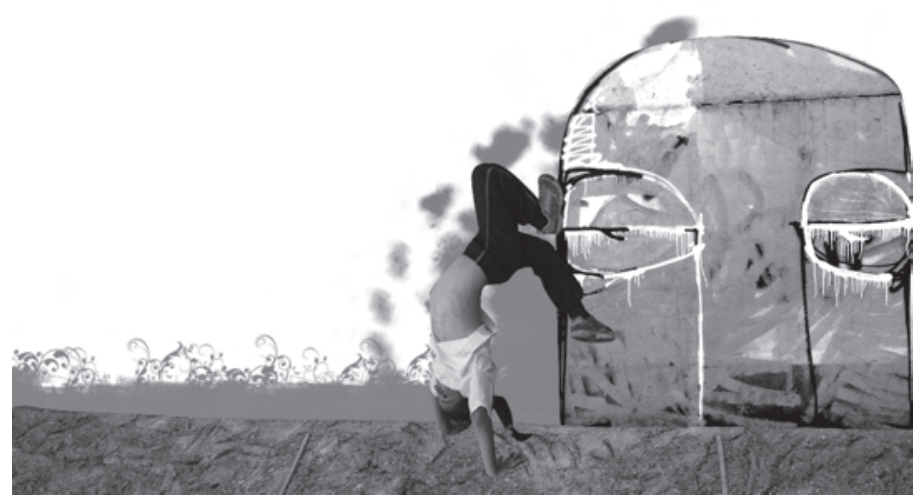

\section{Educación para la}

\section{democracia y la formación política en Colombia: $1980-2006$}

Este es el título del segundo estado del arte, que fue elaborado por Alejandro Peláez y Diego Márquez para la fundación alemana de corte católico Konrad Adenauer, en 2006, y publicado al mismo tiempo que el otro.

Estos autores coinciden con los anteriores en que, en la última década, se ha incrementado notablemente la producción literaria de documentos relacionados con participación política y formación de la ciudadanía. Este fenómeno se nota, sobre todo, en la gran cantidad de trabajos publicados sobre control ciudadano, planeación participativa y competencias ciudadanas. Se puede afirmar que estos temas se han convertido en un renglón importante de la agenda de gobierno del país al momento de definir sus políticas educativas. Por otra parte, gran número de instituciones de diferente naturaleza han dirigido sus objetivos misionales hacia su estudio o promoción (Peláez y Márquez, 2006, 7).

La metodología usada para la elaboración de este estado del arte se basó en un análisis cuantitativo bibliométrico y un análisis cualitativo de contenido. El documento se extiende en la descripción del procedimiento y las herramientas utilizadas antes de adentrarse en el análisis propiamente dicho del corpus.

Consultaron 458 documentos, que organizaron así:

- Publicaciones de organizaciones cuya misión o labor se relacionaban con la educación en democracia y formación política en Colombia. 
- Identificación de fuentes citadas en documentos relevantes para el tema de manera que se determinara el aporte bibliográfico; lo que no logran hacer, pues afirman que muchos de los documentos no tienen bibliografía.

- Establecieron líneas temáticas: metodologías pedagógicas; paz, convivencia y solución de conflictos; debates y definiciones; competencias ciudadanas; derechos humanos desde la perspectiva pedagógica; instituciones democráticas y Constitución; sistema electoral y participación; control ciudadano y experiencias particulares.

El segundo capítulo es especialmente pertinente para este balance ya que se dedica a elaborar un contexto histórico de la educación para la democracia en Colombia. En él se distinguen tres niveles: la coyuntura internacional, el contexto nacional y las políticas educativas adelantadas tanto en la escuela como en las campañas de promoción de la democracia y la ciudadanía llevadas a cabo por el Gobierno Central y los gobiernos locales.

Retomando dos artículos del historiador colombiano Jorge Orlando Melo, afirman que la educación cívica ha sido siempre una preocupación en Colombia y que desde hace casi 200 años los estudiantes han debido aprender, en buena parte como un catecismo que se memoriza, las reglas de conducta cívica. Mientras que las prácticas reales en la escuela o en la sociedad contradecían esas normas o enseñaban en los hechos que quizás no era muy razonable obedecerlas.

Sitúan a la educación cívica y la urbanidad como una necesidad para la construcción de la nación; la aparición en 1910 de la enseñanza de la historia como vehículo para la educación de la ciudadanía y la promoción de la idea de gobierno escolar de la mano de las pedagogías activas en 1930, lo que se vio reforzado por la puesta en práctica de los valores democráticos por medio de la discusión de problemas y la publicación de periódicos. Los autores consideran que esta idea progresista de la educación ciudadana se ve truncada con la instalación de la dictadura de Rojas Pinilla, que según afirman, no estuvo interesada en promover valores democráticos y en su lugar fueron instauradas las cátedras bolivariana y de hispanidad.

Para los autores,

Los planes de estudio relacionados con la cívica en las décadas de los sesenta, setenta y principios de los ochenta, hicieron énfasis en la memorización de las características, funciones y mecanismos de las instituciones políticas, sin ninguna contextualización con las realidades económicas y sociales que rodeaban dichas instituciones. Este enfoque prestaba poca atención al entendimiento de las dinámicas sociales, políticas y económicas; reducía la ciudadanía al mecánico hecho de votar; resaltaba los deberes ciudadanos sobre los derechos y dejaba de lado los intereses políticos de la juventud (Torney-Purta, Rodríguez et al., 1999, citado por los autores).

La reforma curricular de 1984 es ubicada como el momento en que vuelve a tomar fuerza la idea de educación para la democracia y en el que aparecen términos como contexto económico, contexto social, derechos humanos, convivencia, sociedad plural y multicultural.

La nueva Constitución es también, para Peláez y Márquez, el mecanismo que amplió la ciudadanía y el espectro de la democracia, por la precisión de varios conceptos: a) el de derechos, que fue dotado de instrumentos para hacerlos efectivos, b) el concepto de autonomía, materializado en el derecho al libre desarrollo de la personalidad, c) el de participación, con sus respectivos mecanismos de 


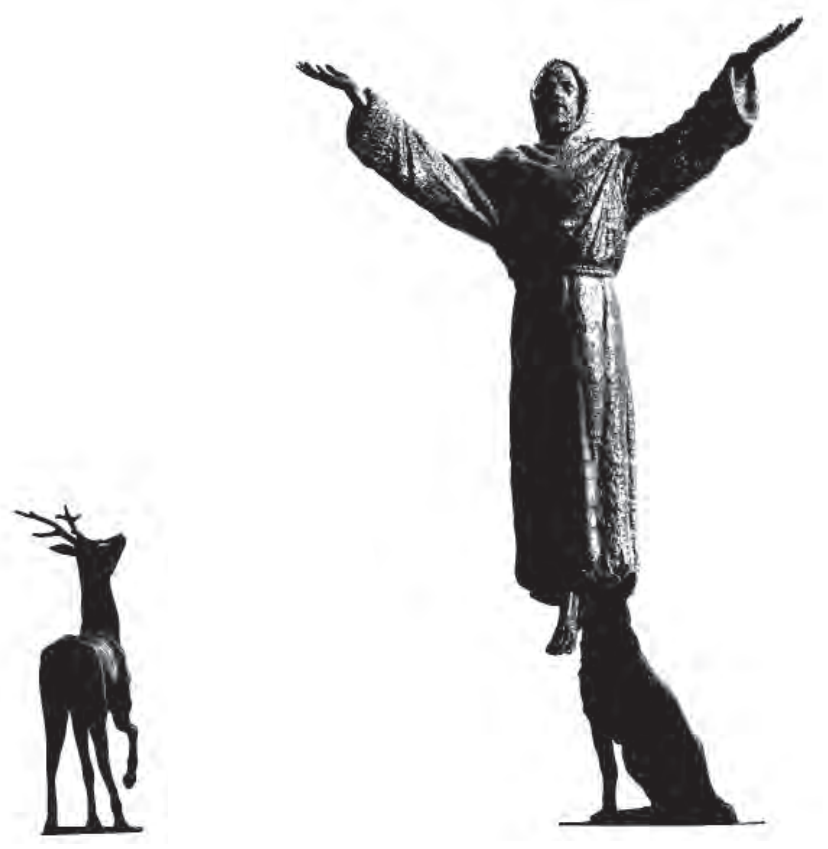

participación política y ciudadana, d) las nociones de pluralismo y diversidad, basadas en la diferencia y la reforma política (Cepeda, 2004, citado por los autores, pp. 23-24). Este suceso es debido, según los autores, a eventos internacionales como el fin de la Guerra Fría, la globalización, el neoliberalismo, y en Colombia, por el conflicto armado, la inestabilidad y la movilización popular.

A partir de la crisis de confianza en las capacidades de los Gobiernos para resolver los problemas de los ciudadanos, destacan la emergencia en los noventa de la sociedad civil representada por el denominado tercer sector y expresado en las Organizaciones No Gubernamentales. Con ellas proliferaron programas y proyectos financiados por agencias de cooperación internacional dedicadas a la promoción de la democracia, a su enseñanza y la formación política.

Los autores identifican dos énfasis adoptados por estas organizaciones dependiendo de sus agendas políticas: una de tendencia de izquierda que centra sus esfuerzos en la movilización de la población a través de la difusión y puesta en práctica de los mecanismos de participación política y jurídica, así como en la defensa de los derechos humanos, y la otra tendencia, vinculada a la industria o financiada por la USAID, que hace énfasis en la lucha contra la corrupción o en la enseñanza de valores y mecanismos democráticos.

Finalmente, hacen alusión al conflicto colombiano como una oportunidad que generó nuevos espacios hacia el desarrollo de la educación para la democracia, referida en especial a la proliferación de publicaciones sobre metodologías para prevenir el conflicto y construir cultura de paz y convivencia.

Frente al carácter de la institución y sus temas de investigación, el estudio encontró que la mayor producción de textos sobre educación democrática y ciudadana emana de las entidades gubernamentales, seguidas de ONG, instituciones educativas (universidades), organismos multilaterales, y por último, entidades privadas (editoriales comerciales).

Quienes más los difunden son las $\mathrm{ONG}$, con temas mayoritariamente sobre instituciones democráticas y Constitución, seguidos por el de derechos humanos, dejando en último lugar los debates y las definiciones teóricas. Quienes menos, son las entidades gubernamentales, que priorizan sus publicaciones en aspectos de control social, dejando también como 
últimas preferencias temáticas las definiciones y las discusiones teóricas. Sin embargo, es importante mencionar el trabajo desarrollado por el desaparecido Instituto Luis Carlos Galán, que parece actuó de manera aislada y desarticulada con las demás entidades estatales.

Dentro de las agencias del Estado, la Alcaldía Mayor de Bogotá tiene un lugar importante en la producción de textos sobre educación para la democracia (35\%), ocupando el último lugar el Ministerio de Educación (8\%).

Como contraste, el sector educativo centra sus publicaciones en los debates y definiciones teóricas, hallándose que la mayor participación se encuentra en cabeza de la Universidad Javeriana y la Universidad de los Andes. En segundo lugar están la Universidad Pedagógica Nacional, la Universidad Distrital y la Universidad Nacional, cuya posición es explicada por los autores así:

La primacía de las universidades privadas en cuanto a volumen y producción se debe básicamente a tres aspectos: la mayor producción de investigaciones y tesis de grado sobre los temas relevantes para este estudio, la existencia de centros de investigación, con líneas investigativas relacionadas con los temas (vgr. Cider e Instituto Pensar), y por último, la fuerte colaboración con el Ministerio de Educación Nacional, en cuanto a trabajos conjuntos.

La producción de los organismos multilaterales como la OEI, la OEA, el BID y la Unicef, ha estado relacionada con la promoción de los valores democráticos y en general con la promoción de una ética pública que sirva como fundamento para un programa de convivencia, paz y resolución de conflictos.
El último lugar en la producción de textos lo ocupan las entidades privadas, quienes se han enfocado a desarrollar el tema de competencias ciudadanas.

Frente al comportamiento de las tendencias editoriales, desde 1980 lo que más se ha publicado, según el estudio, son textos sobre instituciones democráticas y Constitución, seguido en orden descendente por los temas de paz y convivencia, control social, sistema electoral y de participación, metodologías pedagógicas, educación en derechos humanos, competencias ciudadanas, experiencias particulares y, por último, discusiones y definiciones teóricas.

Afirman los autores que antes de 1991 la producción del Gobierno fue muy baja, pero consideran que después de la Posguerra Fría ha aumentado debido al fomento de las competencias ciudadanas, lo que ha promovido el debate, tanto metodológico como teórico, sobre la enseñanza de la educación cívica dentro del esquema de las competencias, derivando, dicen, en el impulso del tema en los otros tipos de instituciones.

Señalan que el tema de paz y convivencia tuvo en 1999 y $2000^{3}$ un significativo incremento; el ámbito de competencias ciudadanas muestra un incremento en el último quinquenio, el de instituciones democráticas y Constitución permanece estable, mientras que la producción de debates y definiciones teóricas muestra una tendencia uniforme con el tiempo, y "parece que no tienen relación con hechos históricos, sino más bien con el giro ordinario de la producción académica", afirmación que no tiene mayores argumentos y es poco clara. 


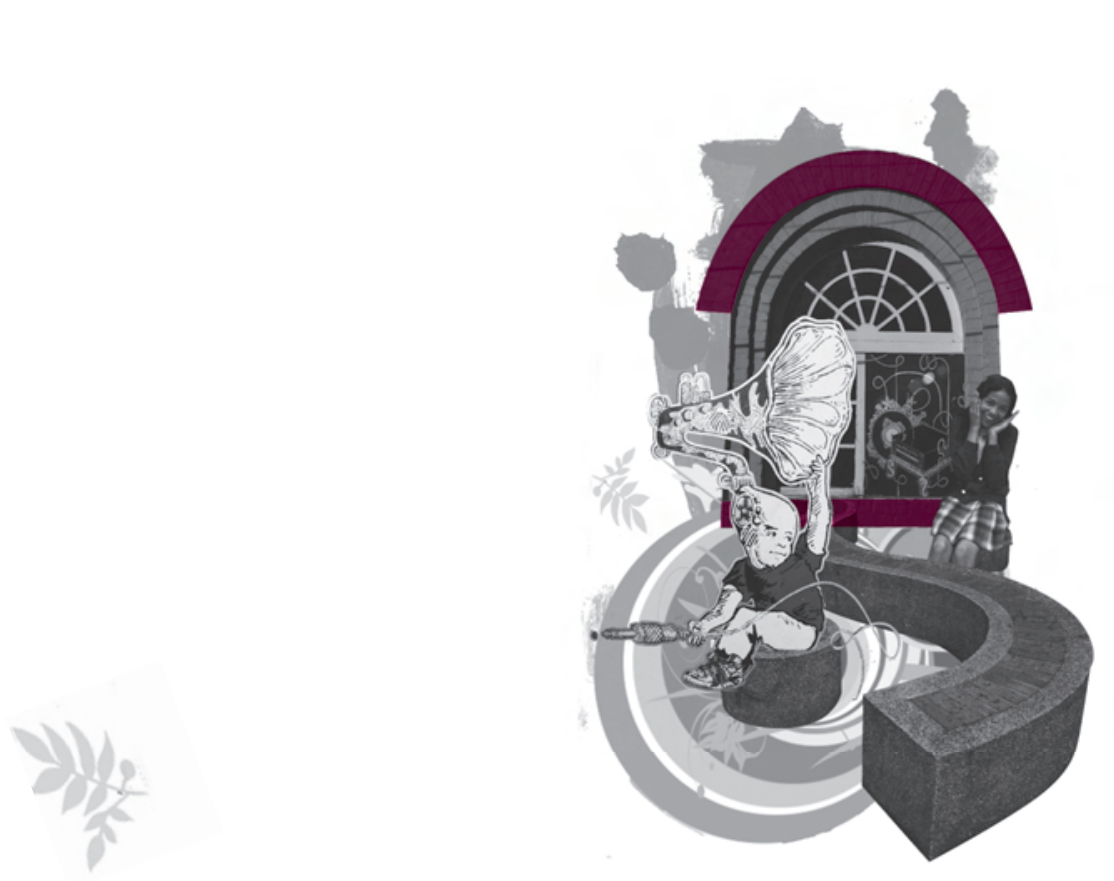

Se concluye que el tema de la educación para la democracia abarca una gran variedad de enfoques y objetivos, evidenciando que hay una gran división entre la educación dentro de las aulas y la formación de la población no escolar.

Para el espacio escolar, se dice que la educación se ha centrado en el suministro de información y últimamente en el tema de competencias. El contenido de los textos se particulariza en el funcionamiento del Estado, los derechos y los deberes ciudadanos, y en investigaciones sobre metodologías o análisis del impacto de programas de este tipo. Se puede afirmar que actualmente, si bien existen diversas metodologías y discusiones sobre el papel que debe desempeñar este tipo de formación, existe un gran consenso en los contenidos y métodos a emplear para educar a los estudiantes para la democracia. Incluso, con la aplicación de las pruebas Saber, resulta posible evaluar (hasta cierto punto) la efectividad del plan de estudio aplicado a los estudiantes.

Para la educación básica, tanto la Constitución del 91 como la Ley 115 son señaladas como acontecimientos importantes que transformaron los contenidos que debían enseñarse en los colegios, pero en ello no profundizan ni identifican estudios que den cuenta de ello. Sólo se menciona la ley de educación y en particular el Decreto $1600,{ }^{4}$ que se refiere al manual de convivencia y el gobierno escolar, así como a las políticas de evaluación y estándares de competencias ciudadanas, como únicas referencias sobre la cuestión de lo que ellos llaman "el avance de la educación democrática y ciudadana en Colombia".

3 La resolución 1600 de 1994 estableció de manera obligatoria incluir dentro de sus Proyectos Educativos Institucionales, el proyecto de Educación para la Democracia. Los autores no consideraron el Decreto 1860, que es la reglamentación que establece mecanismos e instancias de participación ciudadana de la comunidad educativa, como el Gobierno Escolar, el Manual de Convivencia, la Personería Estudiantil, entre otros. 
Desafortunadamente pasan muy rápido las décadas de los sesenta, setenta y ochenta, con una explicación que generaliza lo que fue la educación cívica en la institución escolar, caracterizada, según los autores que referencian, como descontextualizada. Esto podría ser una afirmación ligera si nos detenemos a examinar lo que significó la neoconservadurización de la educación ciudadana en Colombia en un contexto internacional complejo a nivel cultural, económico y político como el de esas décadas. ${ }^{5}$ De la misma manera, no compartimos la conjetura sobre la poca participación del Gobierno en la producción de material sobre educación cívica, democrática o ciudadana antes de 1991, pues la historia de la educación ha demostrado lo contrario. ${ }^{6}$
Los autores dejan en claro que la preocupación de los organismos gubernamentales es el control y plantean un interrogante sobre el papel de la Academia, en especial de la Universidad pública. Desafortunadamente no asumen la institución escolar como institución de saber y de producción de discurso, lo que el anterior estado del arte sí recoge.

4 Ver Álvarez, Alejandro, Las Ciencias Sociales en el currículo escolar. Colombia, 1930-1960, tesis doctoral, dir. Gabriela Ossenbach Sauter, Madrid, Departamento de Historia de la Educación y Educación Comparada, Facultad de Educación, UNED, 2007; Martínez Boom, Alberto, De la escuela expansiva a la escuela competitiva. Dos modos de modernización en América Latina, Barcelona: Antrhopos, 2004.

5 Ver: Herrera, Martha; Pinilla Alexis, Suaza, Luz Marina, La identidad nacional en los textos escolares de ciencias sociales Colombia 1900-1950, Bogotá, Universidad Pedagógica Nacional, 2003; "La enseñanza de la geografía y de las matemáticas en la década del treinta”, en Revista Educación y Pedagogía, vol. XIII, nº 29, Medellín, Universidad de Antioquia, 2007, pp. 113-128 "Las Ciencias Sociales en el currículo escolar. Colombia, 1930-1960", tesis doctoral, dir. Gabriela Ossenbach Sauter, Madrid, Departamento de Historia de la Educación y Educación Comparada, Facultad de Educación, UNED; Quiceno, H., "La clase y el libro didáctico", en Reflexiones pedagógicas, n 1, Cali, 1980, pp. 11-17; "El manual escolar: pedagogía y formas narrativas", en revista Educación y Pedagogía, Vol. XIII, no 29, Medellín, Universidad de Antioquia, 2001, pp. 53-67; "Manuales, ensayos y crónicas en la educación en Colombia, 1900-1930”, en Conde Calderón J. et al. (comps.), Nación, educación, Universidad y manuales escolares en Colombia. Tendencias historiográficas contemporáneas. IV Coloquio Colombiano de Historia de la Educación, Barranquilla, Universidad del Atlántico, 2002, pp. 449-460; Sáenz J., Saldarriaga O. y Ospina A., Mirar la infancia: pedagogía, moral y modernidad en Colombia, 1903-1946, Medellín, Colciencias / Ediciones Foro Nacional por Colombia / Ediciones UniAndes / Editorial Universidad de Antioquia, 2 vols., 1997; Saldarriaga O., "Los manuales de filosofía neotomista en la educación secundaria colombiana. Una matriz pedagógica del ‘sentido común', 1865-1965”, ponencia presentada en el VI Congreso Iberoamericano de Historia de la Educación Latinoamericana, San Luis Potosí (México), 2003; Alarcón, Luis Alfonso, "Entre Dios y la patria: la formación del ciudadano en Colombia a través de los manuales escolares", ponencia presentada en el VIII Congreso Iberoamericano de Historia de la Educación Latinoamericana, Buenos Aires, 2007. 


\section{A manera de cierre}

Los dos estudios coinciden en mirar la ley como un a priori de las prácticas de formación ciudadana y democrática y confirman la primacía de su carácter empírico, mostrando que el tema es más de preocupación por acciones de intervención socioeducativa que de reflexiones académicas. La referencia a la nueva Constitución se reitera como evento que produce un giro en las prácticas de la educación ciudadana y que invita a mirar cómo es ese giro, más allá de asumir que con ella entramos a una ciudadanía plena. Del mismo modo, ambos estudios se refieren al tema de la evaluación de los aprendizajes ciudadanos aunque desde valoraciones opuestas, ubicándolo como una nueva práctica que desplaza quizá la formativa.

Es importante señalar que lo común en lo que analizan estos estados del arte es la búsqueda de respuestas sobre si la escuela ha cumplido o no con la tarea de educar a los ciudadanos en un orden social particular. El afán por buscar una relación de coherencia entre lo ideal y lo real remite a un análisis en el que predomina una visión evolutiva y progresista que muestra sucesivos fracasos que han tratado de ser superados por modelos cada vez más democráticos de "educación ciudadana" y en los que la discusión es si el modelo actual es mejor y cómo debe operar.

De igual manera, parece haber un consenso en dos grandes momentos de la "educación ciudadana" y que coinciden con dos modalidades políticas: el ciudadano nacional situado en el discurso de la nación, el ciudadano cultural situado en los discursos de la diversidad. Estos dos lugares de enunciación legitiman el cambio de una imagen construida sobre una ciudadanía que ya no se quiere por ser homogeneizante y excluyente, por

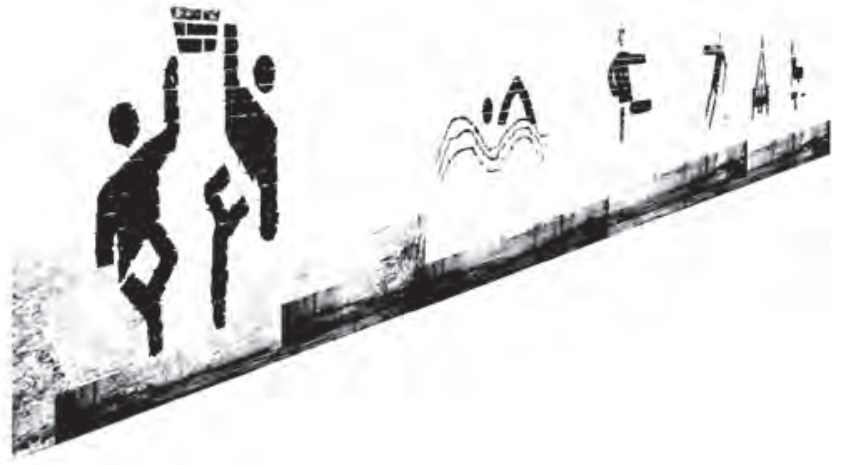

otra deseada, que sería una ciudadanía incluyente y diferenciada.

También muestran la multiplicación y multiplicidad de objetos de discurso sobre la ciudadanía que se deslocalizan de la teoría del Derecho reinante en textos escolares, planes de estudio y currículos hasta la década de los setenta, para dar lugar a otras nociones provenientes de diversos saberes, como la psicología moral, la filosofía moral, las ciencias políticas, la sociología y la antropología.

Finalmente, se debe reconocer que los dos estudios amplían de manera complementaria una significativa masa documental que abarca instituciones y organizaciones que elaboran discursos sobre educación ciudadana, y muestran la pluralidad de temas abordados y los énfasis según los intereses de quien los produce, constituyéndose en un importante aporte para quienes nos interesa este tema. 


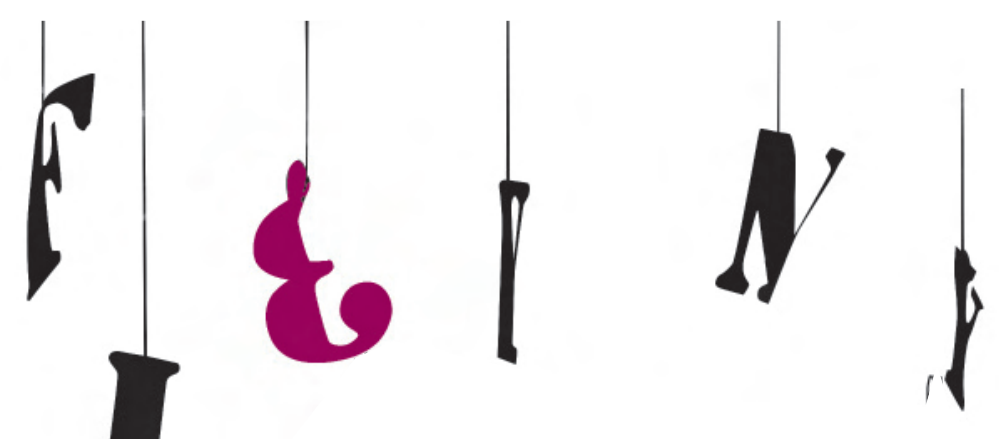

\section{Bibliografía}

Cortés Salcedo, R. A. “Educación ciudadana y escuela: balance historiográfico, Bogotá 1985-2007”, ponencia presentada al XIV Congreso Colombiano de Historia, Tunja, Universidad Pedagógica y Tecnológica de Colombia, 2008.

García, R.; Serna, A. Dimensiones críticas de lo ciudadano, Bogotá, Universidad Distrital, 2002.

Peláez, A.; Márquez, D. Educación para la democracia y la formación política en Colombia: 1980-2006. Bogotá, Fundación Konrad Adenauer, 2006.

Pinilla, A.; Torres, J. C. De la educación democrática a la "educación ciudadana": una década de incertidumbres, Bogotá, Universidad Pedagógica Nacional - IDEP, 2006. 